\title{
Developing an electronic record keeping system at a paediatric clinic in Colombo South Teaching Hospital, Sri Lanka
}

\author{
D S Wijesekara ${ }^{1}$, *P L S Peiris ${ }^{2}$, D S Fernando ${ }^{2}$, T D N Palliyaguru ${ }^{2}$, W A D N Fonseka ${ }^{2}$
}

Sri Lanka Journal of Child Health, 2020; 49(2): 116-124

\begin{abstract}
Introduction: Paediatric multi-disciplinary team (MDT) clinic at Colombo South Teaching Hospital is designed for patient-centred management of paediatric neurological disorders. This clinic lacks an electronic database management system (EDBMS). Open source technology through Google and AppSheet ${ }^{\circledR}$ provides tools to create E-DBMS.
\end{abstract}

Objectives: To design a user-friendly, secured electronic DBMS for record keeping, audit, data analysis and research purposes and pilot testing it.

Method: A new Google account was created for the MDT clinic. Google spreadsheets ${ }^{\circledR}$ were used to design the data tables and were linked to AppSheet ${ }^{\circledR}$ software to generate graphical user interface of the mobile app of the database. Appearance and features of the App were designed through options provided by the AppSheet ${ }^{\circledR}$ without writing codes using computer language. Once the app was ready, email address and password were shared among the authorized team members and the mobile app was installed into their phones.

Results: This app enabled the team members to add, edit and view data of MDT patients. Telephone calls could be taken and patients' locations could be accessed through the app. Since the database was accessible through phones, patients' past records could be reviewed and new records could be entered while conducting the clinic. Success of the therapies given to patients can be quantified by motor skills score and Alberta Infantile Motor Scale (AIMS) centile analysis. These spreadsheets could be linked to the Statistical Package for Social Sciences (SPSS)

${ }^{1}$ Senior Lecturer and Consultant Paediatric Neurologist, ${ }^{2}$ Demonstrator, Department of Paediatrics, Faculty of Medical Sciences, University of Sri Jayewardenepura,

*Correspondence: lashansanojpeiris@gmail.com

orcid.org/ 0000-0001-8431-3861

(Received on 28 June 2019: Accepted after revision on 16 August 2019)

The authors declare that there are no conflicts of interest

Personal funding was used for the project.

Open Access Article published under the Creative

Commons Attribution CC-BY (C) (†) License software to analyse data for research purposes. Even though this provides adequate confidentiality and security to data, all the authorized team members who share the app have an unrestricted access to add, edit or remove data.

Conclusions: Electronic DBMS could be designed practically via AppSheet ${ }^{\circledR}$, which is an effective tool to maintain and to retrieve patient records and for research purposes.

DOI: http://dx.doi.org/10.4038/sljch.v49i2.8958

(Keywords: Database management system, Google services, AppSheet, paediatric neurology MDT clinic)

\section{Introduction}

Paediatric multi-disciplinary team (MDT) clinic at Colombo South Teaching Hospital (CSTH) is a subspecialty clinic which was started in the recent past by the Professorial Paediatric Unit of the University of Sri Jayewardenepura. This clinic was specially designed for patient-centred management of paediatric neurological and developmental disorders with input from the paediatric neurologist, other paediatric medical officers, physiotherapists, occupational therapists, speech therapists, orthotics and prosthesis specialists, and, if needed, child psychiatrists etc. Patients are referred from paediatric units of the hospital as well as other government hospitals. Patients are assessed and treatments are started and adjusted at MDT visits. Improvement of the clinical condition is assessed at each MDT clinic visit. Recently, a new programme was commenced to incorporate neonates to the clinic with risk factors for future development of developmental delay and neurological deficits and start early interventions to improve the outcome of these children, and these early interventions have immensely helped in the developmental outcomes of the children.

Health sector in Sri Lanka lacks Clinical Database Management (CDM), which is an important element of patient care. We do not have a credible system to store and retrieve clinical data. Doctors and medical specialists in general, document and store data on paper predisposed to illegibility, loss or irretrievability. Dedicated hospital based electronic medical record (EMR) systems or database management systems (DBMS) are available in 
developed countries and some of the hospitals in Sri Lanka to overcome this issue. However, these too have an inherent deficiency of selecting suitable patients for clinical studies that require researchers to manually read through data tables and reports to filter and gather data.

In a developing country like Sri Lanka, creating and initiating a networked DBMS for CDM in hospitals poses significant budgetary constraints. A good DBMS is important for assessing improvement of patients as well as promoting our doctors to make advancement in medical research. Additionally, it will generate valid medical data from our part of the world accessible for international researches and paradigm shifting hallmark studies.

Paediatric MDT clinic at Professorial Paediatric Unit, CSTH has failed to develop previously and maintain a DBMS due to logistics issues. Lacking a DBMS caused inability to assess the success of the therapies for individual patients as well as the effectiveness of the whole patient cohort. When required, other team members could not access data due to lack of a clinical database. We identified some important requirements which made it difficult to maintain an electronic database for the purpose of audit and research as user-friendly interface to enter data into database, irretrievability of entered data for statistical analysis and dedicated personnel to maintain the database. Our aim is to find effective solutions for these three obstacles.

We propose a systematic approach to address these issues and present a solution - A cloud-based database system, with a custom made graphical user interface (GUI), that any member can access from their own mobile phone at any time, powered by AppSheet (AppSheet Seattle, Washington, USA), Google Forms ${ }^{\circledR}$ and Google Sheets ${ }^{\circledR}$ (Google, Mountain View, California, USA) that gets instantly updated upon data entry and store data on Google Drive ${ }^{\circledR}$ in a retrievable format for data analysis. This paper gives an easy understanding on how to create a DBMS with AppSheet and Google Sheets ${ }^{\circledR}$ in a step wise manner.

\section{Objectives}

To design a user-friendly, secured electronic DBMS for record keeping, audit, data analysis and research purposes and pilot testing it

\section{Method}

This section will contain a step-by-step guide to create a custom made mobile app to enter patient data on a mobile phone using Google services and AppSheet platform.
Step 1: Establish a Google Account:

It is advisable to have a separate Google account for the management of the DBMS, so that the password can be shared among the members of your team who are certified to handle data. A Google account could be created by visiting http://account.google .com/.

Step 2: Creating a Google spreadsheet based on the necessary data fields:

Once the Google account is created, log on to it and access Google Apps grid on top right hand corner of the screen (Figure 1A) and select Google drive from it (Figure 1B).
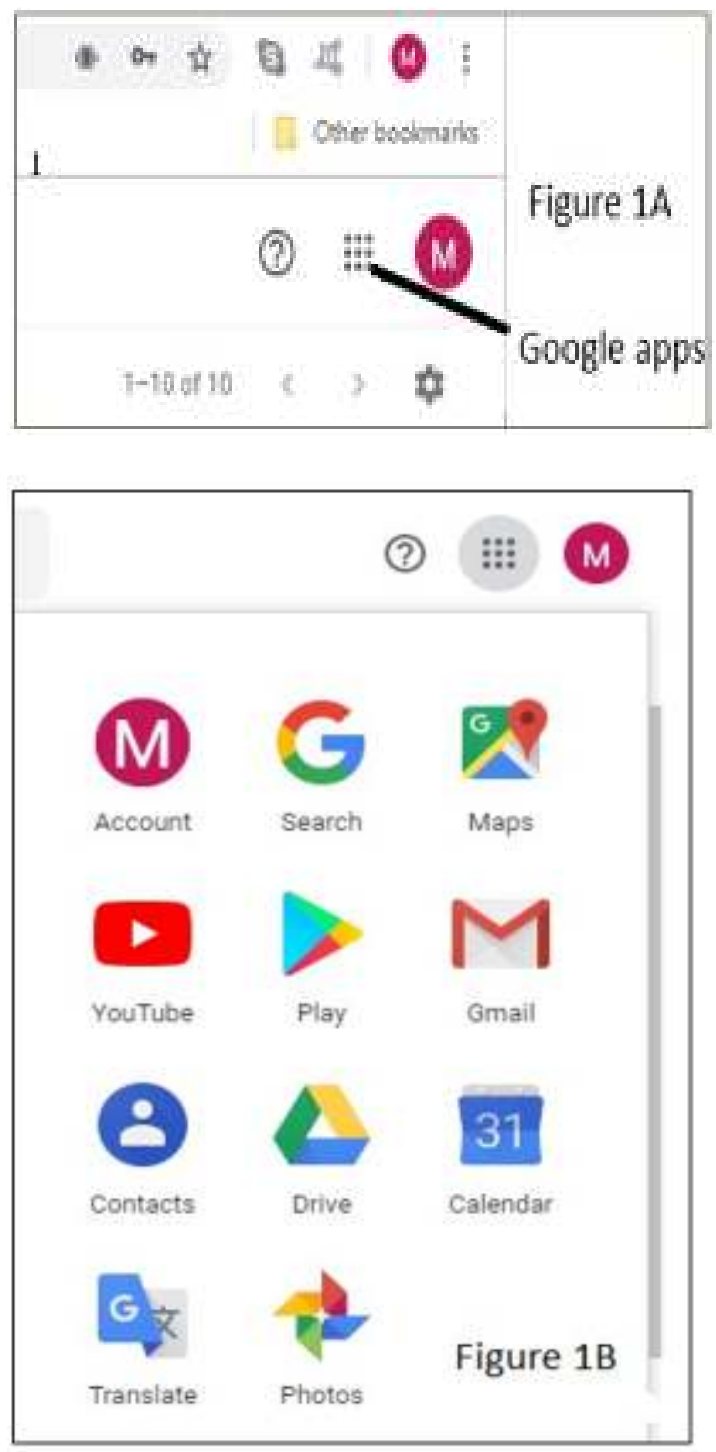

Once the Google Drive page opens, click on $+\mathrm{New}$ button on the top left hand corner (Figure 2A) and select Google Sheets and then, blank spreadsheet. 


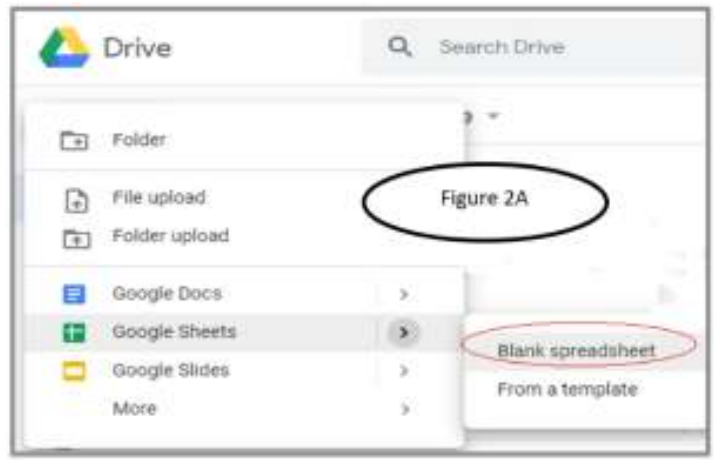

The standard blank spreadsheet will appear as shown below. (Figure 3)

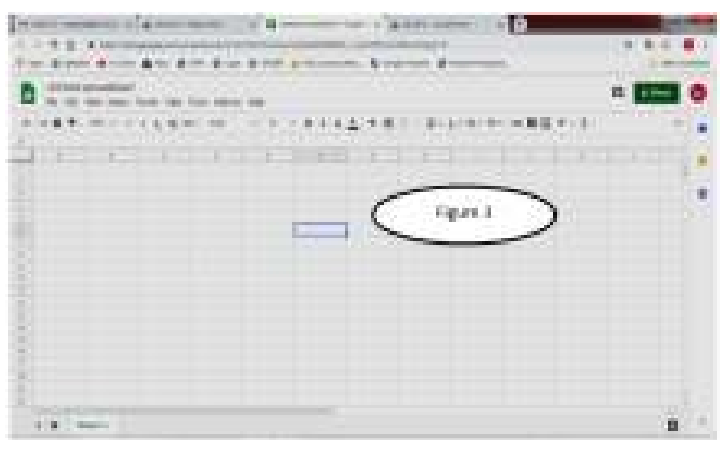

Rename the Google spreadsheet. Then, Design the data tables which are needed to be included in your database within the Google spreadsheet. Use the "+" button on the bottom left hand corner in the Google spreadsheet window to create several data sheets (data tables) within your spreadsheet. Designing these tables is the key step to create the DBMS as well as Mobile App of the database. The data sheets/tables you will be designing this way will be the data fields of the App you will be creating later.

Step 3: Link the Google Spreadsheet to AppSheet to create the Graphical User Interface of the mobile app.

Upon finalizing the Google spreadsheet, it should then be linked to AppSheet software to create the Graphical user interface of the mobile app. Go to https://www.appsheet.com/ to get the free version of the AppSheet software. (Figure 4) Then, click the button "Start for free".

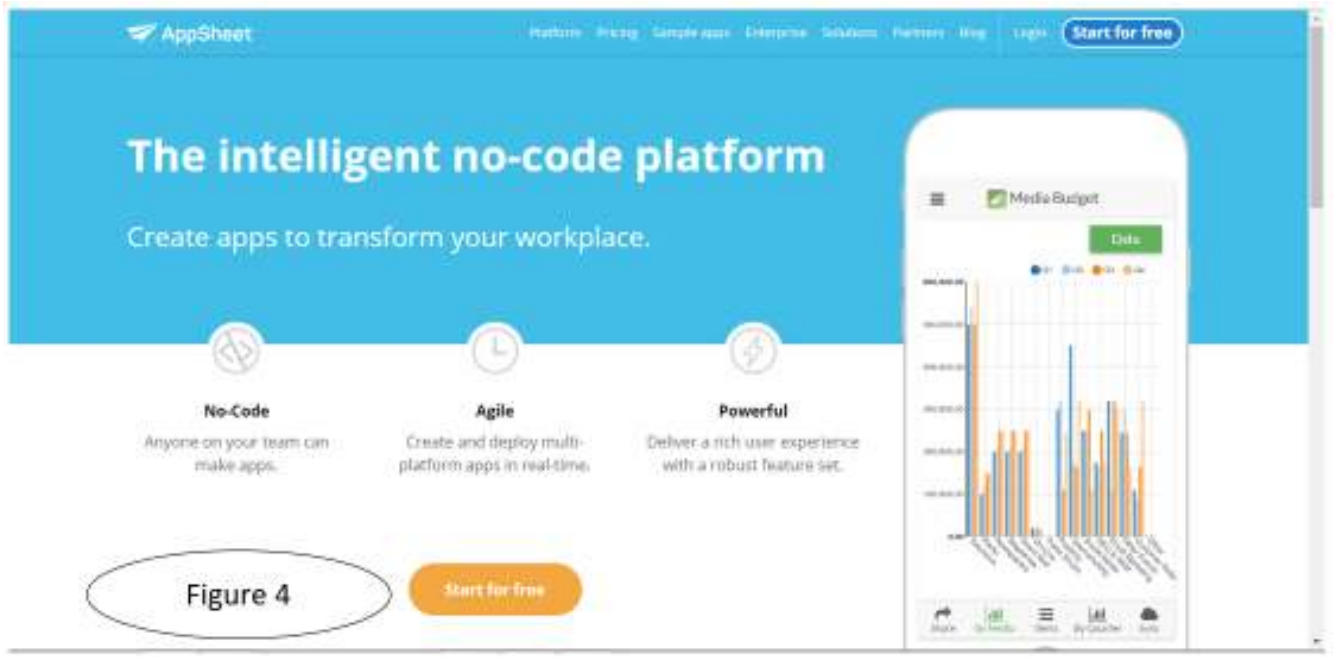

Then, Select Google sheets and forms. Then Sign in to the newly created Google account (As mentioned in step 1) with the e-mail address and password. Then, Click "Allow" giving access to AppSheet to use the Google account (Figure 5).

Then, AppSheet Window will appear and select "Make a New App". Once you click that, three options will be provided and select "With Your Own data" (Figures 6 and 7).

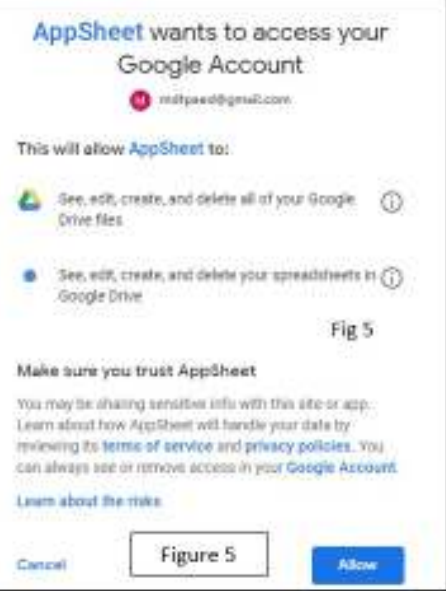




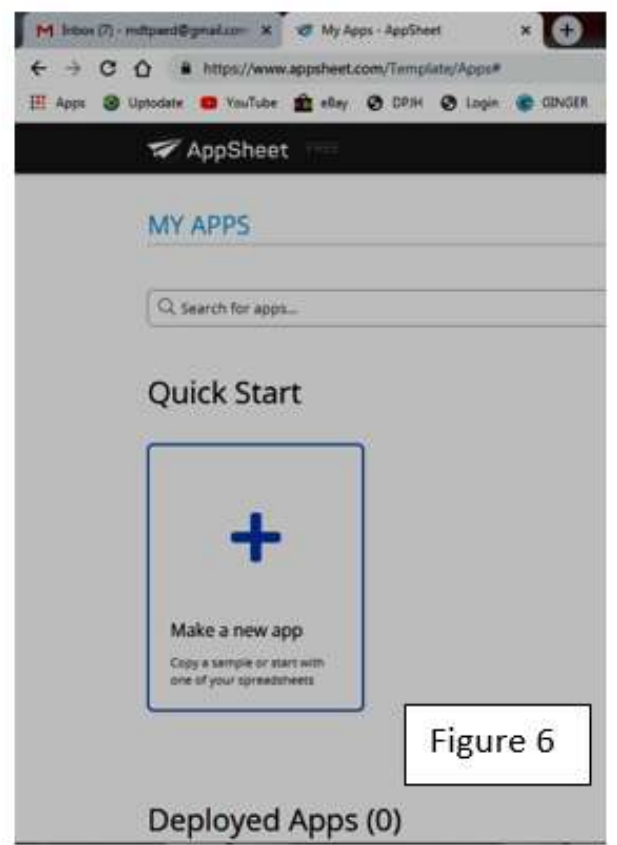

Deployed Apps (0)

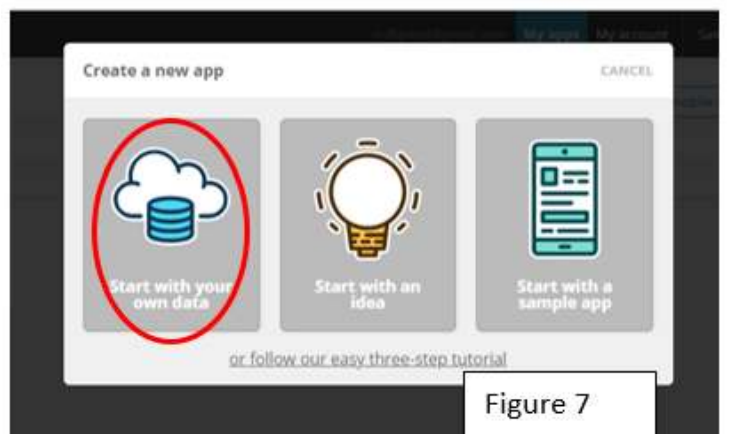

Then Give a name to your app and select any category from "Set Up your new app" window appeared when you do the above step.

Then click "Choose your data". Once you have done this, a new window will appear to select the spreadsheet. Select the Google spreadsheet which was created earlier.

Then, GUI of the new app will appear in this AppSheet window as below (Figure 8).

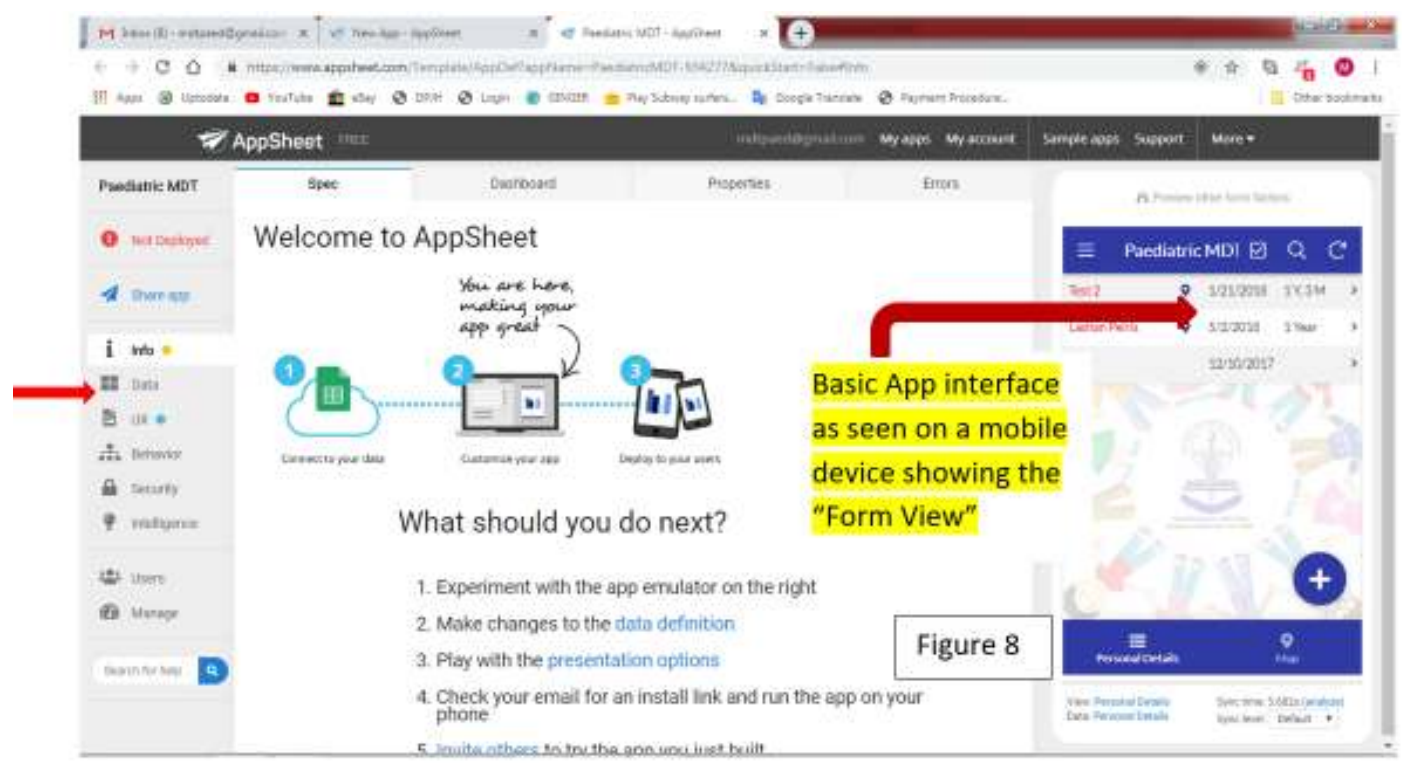

Step 4: Modify the GUI of the Mobile App using AppSheet

Then, Make use of the various options available such as "Data", "UX" to build up the GUI of the newly created app. You can use "Data" option, to add new tables to the database.

- Select "Data" [Red Arrow in Figure 8].

- Click "+ Add New Table"

- Select the Google spreadsheet from the drop down menu in the "Source ID "field.
- Select the Table/Sheet from the "Worksheet name/Qualifier" drop down menu.

- Click "Add this table" [Figure 9]

Once you add a new table, select the table and click "Updates, Adds, Deletes" from "Are updates allowed" menu as in Figure 9.

Always Click "Save" after each change which you have done in the AppSheet. 


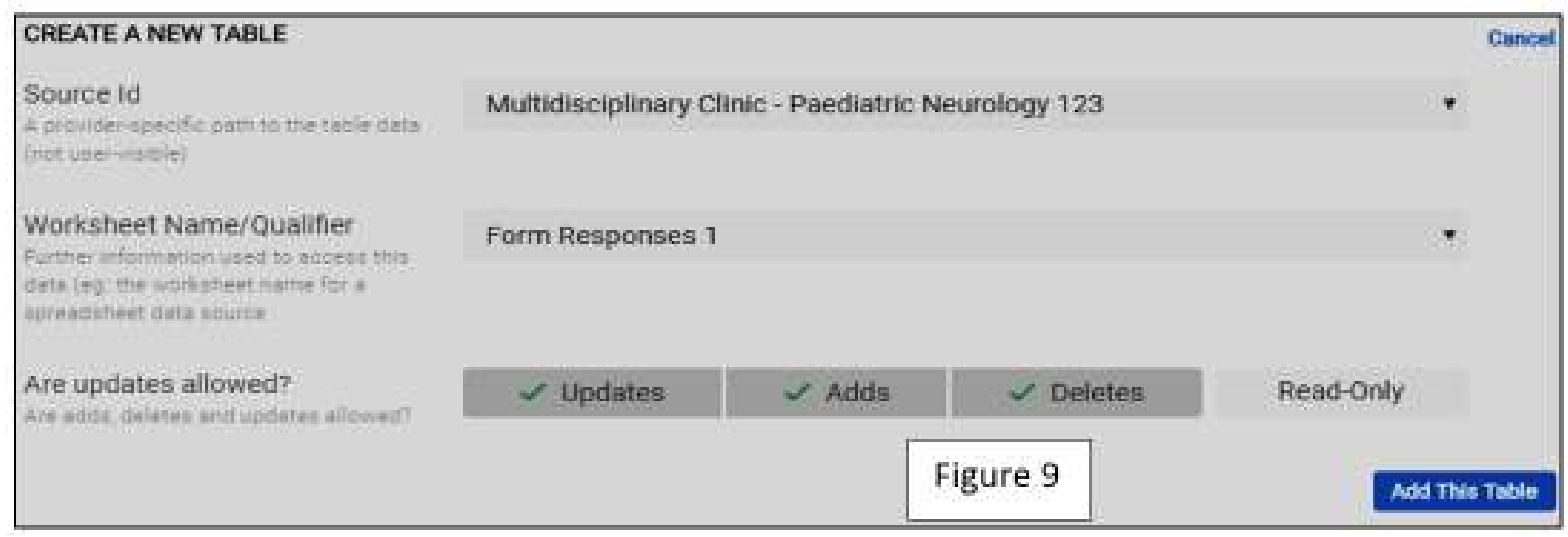

The AppSheet web based platform allows the users to change the external appearance of the app by adding colour themes, custom app logos, launch images and background images. You can explore the "UX", "Data" and "Behavior" options on AppSheet to further customize the app to your own taste. App formulas, Slices, References between Tables and incorporating them to the app would further enhance the capabilities of your app, but it is beyond the scope of this paper.

The AppSheet web based platform will open up in a new tab on your web browser with a basic mobile app interface displayed on the right hand side. [Figure 8]

The present app interface on Figure 8 shows the "Form View" of the app. It is based on the Google Spreadsheet that you created earlier. You can click the "+" purple button to add a new data. The added data records of individual patients will appear as in figure 8 . You can see the data by clicking the data records name in form view of the app.

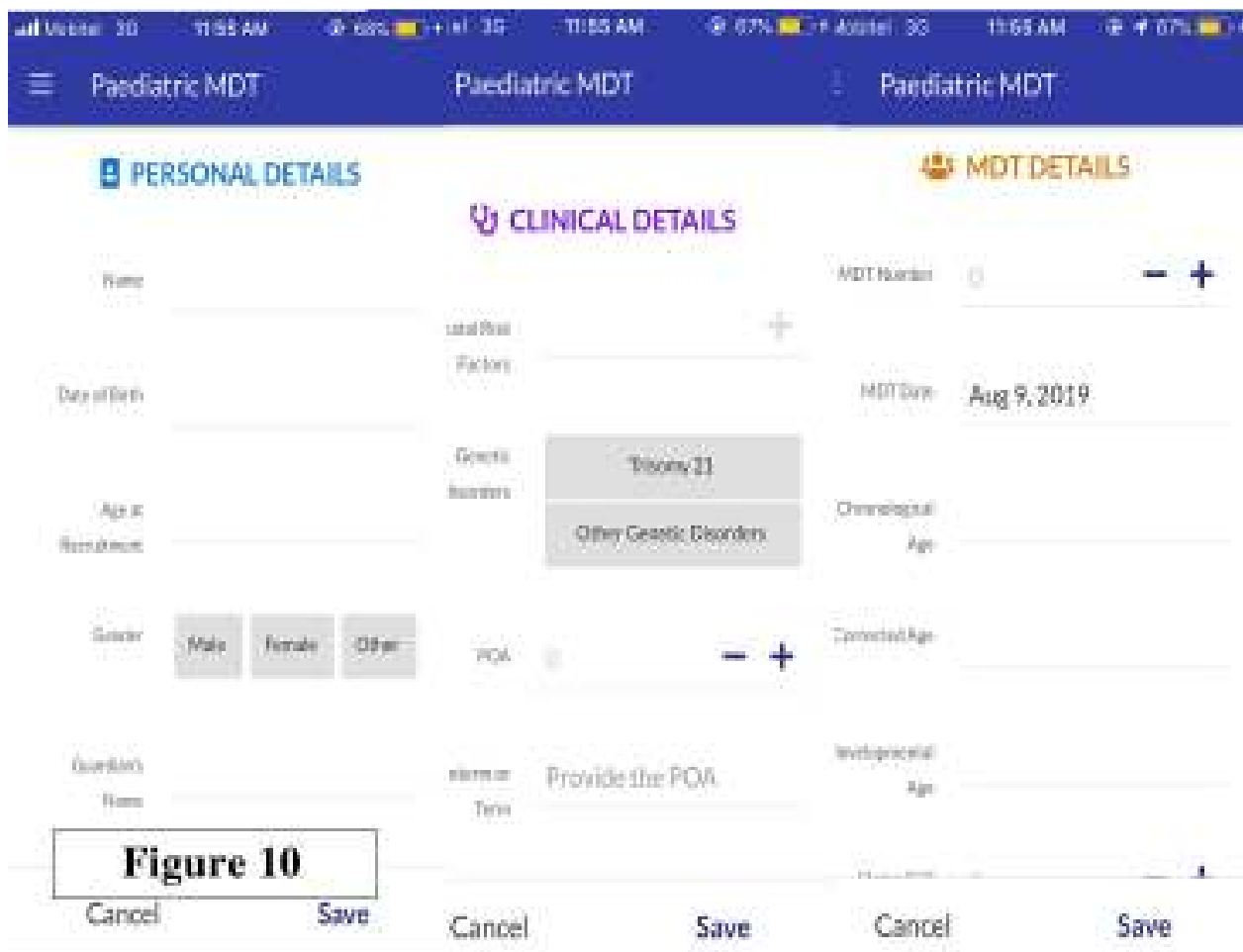

This form view shows "Name" - as a short text field, "Age at recruitment" as a numeric field, "Gender" as a multiple choice question etc. Once you entered data, click "Save". When you click the "Clinical detail" tab, you can enter data to clinical details table. (Figure 11)
Upon populating the spreadsheet which is saved in the Google Drive, user can access it anytime for data analysis. The Google spreadsheet can be connected to SPSS through Microsoft Excel. 
Step 5: Finalizing and launching the mobile app among authorized team members

Once the app is ready to be shared with your team, head over to "Users" section of the AppSheet web platform and enter email addresses of the team members and send invites. When the team members receive the invites to their corresponding email accounts, they should click the link on the invite. This will prompt them to install the AppSheet App on their phones from the App Store (iOS) or Play Store (Android) on their devices. Once it is installed, open the AppSheet app on their phones and log in using their own Google email address or the Google email address that was created earlier to create the database. The team members will now be prompted to install your database apps shortcut to the home screen of their mobile device.

Maintaining the database in the cloud can be considered a limitation as it requires an internet connection always, although it is what makes this approach more secure and available across multiple devices at the same time.

\section{Results}

Electronic Database Management System (eDBMS) and custom-made mobile app are created for the paediatric MDT clinic by utilizing the above mentioned method. The database and mobile app was launched from the beginning of April 2019. Email address and password of the Google account were shared among the authorized team members. Data entry into the MDT clinic database was started through the mobile app as a pilot study.

Appearance of the front page and records view are shown in figures 12 and 13.

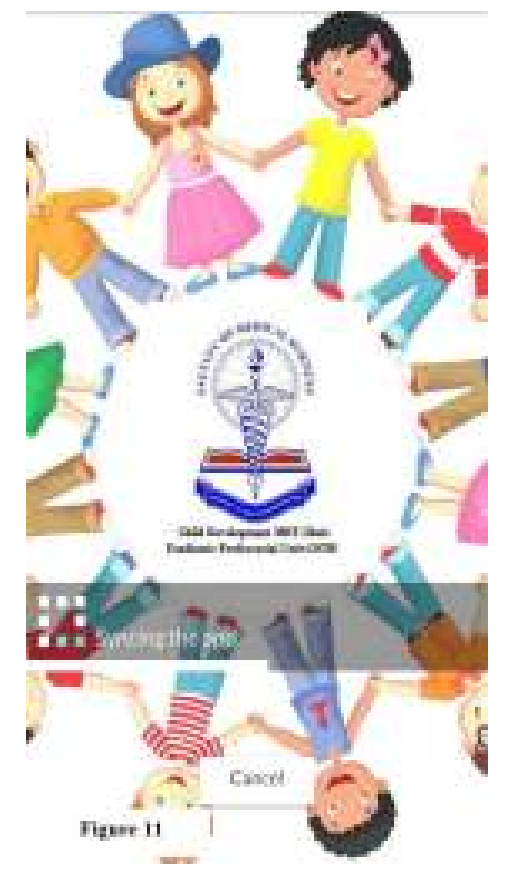

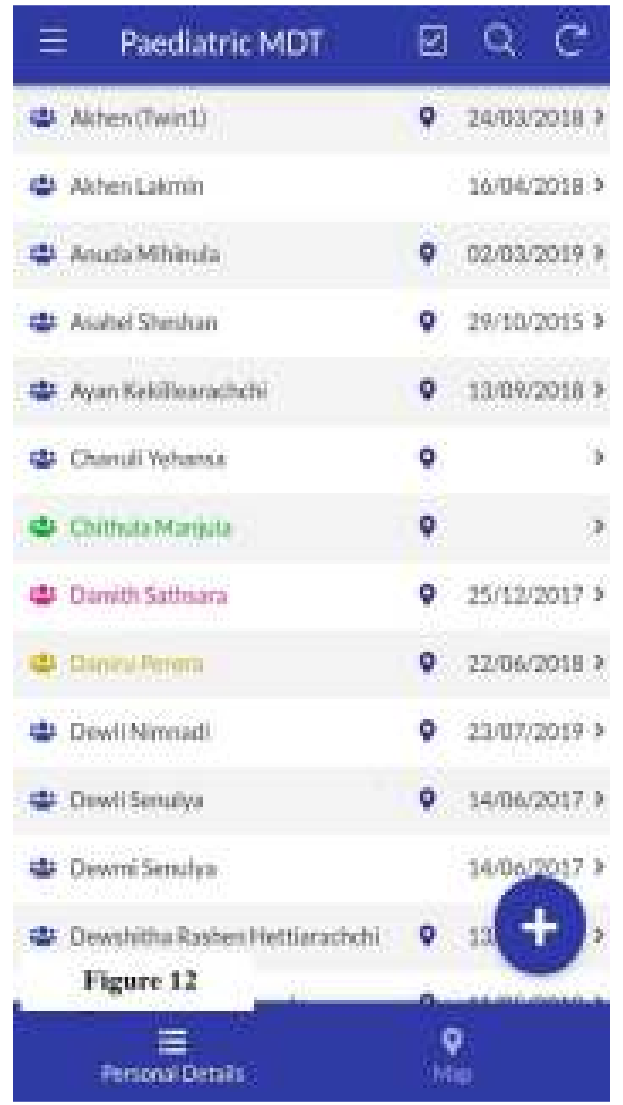

Features given by the Database/Mobile App

Personal details table/form is the first form to be filled in the database and it contains data fields such as Name of the patient, date of birth, age at recruitment, gender, guardian's name, contact number, address, and disease category of the patient (Early interventions, Developmental delay/Cerebral palsy, Neuromuscular disorders, Seizure disorders, Genetic and chromosomal anomalies and other).

Telephone calls can directly be taken through the app after entering patients' phone number into the database. After entering patients' addresses into the database, the mobile app allows to find the patients' home locations through Google maps.

Records view (Figure 13) gives the list of records with patients' names appearing in different colours for separate disease categories e.g. black/navy blue: early intervention, green: cerebral palsy, pink: genetic disorders, orange: neuromuscular disorders, red: seizure disorders, brown: other category etc.

Clinical details table/form is the second form to be filled and it contains data fields as neonatal risk factors as a drop down menu, Period of amenorrhea (POA), details of brain imaging, eye referral and ear, nose and throat (ENT) referrals, 2D echocardiography, hip joint X-rays and/or ultrasound scan findings of the hip joint, if relevant, and other investigation findings. 
When the POA is entered, app automatically gives the categories of maturity as term, late preterm, early preterm and extreme preterm. Photos of the investigation reports can be taken through the mobile phones and uploaded into the database directly.

When the personal and clinical details forms are filled for one individual patient, another form appears to enter MDT details. Records of all MDT visits of one individual patient can be entered into the database. MDT details form/table contains data fields such as date of the MDT visit, chronological age, corrected age and developmental age of the patient at respective MDT visit, motor skill assessment score, Alberta Infant Motor Skills (AIMS) scores and AIMS centiles (Less than $5^{\text {th }}, 5^{\text {th }}$ to $10^{\text {th }}, 11^{\text {th }}$ to $25^{\text {th }}, 26^{\text {th }}$ to $50^{\text {th }}, 51$ th to $75^{\text {th }}, 76^{\text {th }}$ to $90^{\text {th }}$, more than $90^{\text {th }}$ ), details of occupational therapy, speech therapy and MDT decisions. Photographs of occupational therapy, speech therapy and MDT decisions records can be taken from the mobile phone and uploaded directly into the database.

This mobile app enabled the authorized team members to add, edit and view data of MDT patients while conducting the clinic. Success of the therapies given to patients can be quantified by motor skills score and AIMS centile analysis.

Google spreadsheet which contains the entered data can be converted into Microsoft Excel and then, converted again into SPSS software for statistical analysis of the data.

\section{Results generated through analysis by SPSS version 17.0 \\ Demographic Distribution}

After implementing the database from April 2019 as a pilot study, data of 66 patients were entered into the database. Among them 46 (69.7\%) were males and $20(30.3 \%)$ were females. Out of the 66 patients, $42(63.6 \%)$ were registered for early intervention for at risk neonates, $11(16.7 \%)$ were cerebral palsy patients and 9 (13.6\%) were Down syndrome patients. Other categories were neuro-muscular disorders and seizures (Table 1). Out of entered patients, $47(71.2 \%)$ were recruited at birth (Table 2).
Table 1

Categories of patients $(n=66)$

\begin{tabular}{|c|c|}
\hline Category & n (\%) \\
\hline Early intervention & $42(63.6)$ \\
\hline Cerebral palsy/ development delay & $11(16.7)$ \\
\hline $\begin{array}{l}\text { Genetic defect/ chromosomal } \\
\text { disorder }\end{array}$ & $09(13.6)$ \\
\hline Neuromuscular disorder & $02(03.0)$ \\
\hline Seizure disorder & $01(01.5)$ \\
\hline Other & $01(01.5)$ \\
\hline
\end{tabular}

Table 2

Period of recruitment to MDT clinic $(n=66)$

\begin{tabular}{|l|c|}
\hline Period of recruitment & n (\%) \\
\hline At birth & $47(71.2)$ \\
\hline After birth within one year & $05(07.6)$ \\
\hline 1-2 years & $08(12.1)$ \\
\hline After 2 years & $06(09.1)$ \\
\hline
\end{tabular}

Majority of the patients were residing in the Colombo district and were from the Piliyandala (22.7\%) and Dehiwala (19.7\%) Medical Officer of Health $(\mathrm{MOH})$ areas. Among the patients who were recruited 36 were premature and majority of them were early preterm patients (Table 3 ).

Table 3

Maturity at birth $(n=66)$

\begin{tabular}{|l|c|}
\hline \multicolumn{1}{|c|}{ Maturity } & n (\%) \\
\hline Term $(\geq 37+0$ weeks) & $30(45.5)$ \\
\hline Late preterm ( 33+0 to 36+6 weeks) & $13(19.7)$ \\
\hline Early preterm (28+0 to 32+6 weeks) & $21(31.8)$ \\
\hline Extreme preterm (<28+0 weeks) & $02(03.0)$ \\
\hline
\end{tabular}

Table 4

Usage of imaging studies

\begin{tabular}{|l|l|}
\hline Imaging Modality & $\mathbf{n ~ ( \% )}$ \\
\hline Ultrasound scan of brain & $50(75.8)$ \\
\hline Computed tomography of brain & $08(12.1)$ \\
\hline Magnetic resonance imaging of brain & $17(25.8)$ \\
\hline 2 or more modalities & $14(21.2)$ \\
\hline
\end{tabular}

Out of the 66 patients, 59 patients have undergone some kind of brain imaging (Table 4). Brain ultrasound scan (USS) has been done in 50 patients and abnormal brain USS were detected in $38.3 \%$. Commonest abnormalities encountered were cordothalamic groove cysts and haematomas. For percentages of the patients who have undergone other brain imaging, refer table 5 . 
Table 5: Frequencies of imaging studies $(n=66)$

\begin{tabular}{|l|c|c|c|}
\hline \multicolumn{1}{|c|}{ Imaging modality } & $\begin{array}{c}\text { Normal Findings } \\
\mathbf{n}(\mathbf{\%})\end{array}$ & $\begin{array}{c}\text { Abnormal findings } \\
\mathbf{n}(\mathbf{\%})\end{array}$ & $\begin{array}{c}\text { Not done } \\
\mathbf{n}(\mathbf{\%})\end{array}$ \\
\hline Ultrasound scan of brain & $29(43.9)$ & $18(27.3)$ & $19(28.8)$ \\
\hline Computed tomography of brain & $02(03.0)$ & $05(07.6)$ & $59(89.4)$ \\
\hline Magnetic resonance imaging of brain & $04(06.1)$ & $11(16.7)$ & $51(77.3)$ \\
\hline
\end{tabular}

Frequencies of the neonatal/perinatal risk factors for future developmental/neurological abnormalities were calculated through SPSS and majority (42) patients have had neonatal sepsis. Other common risk factors identified were neonatal jaundice, intrauterine growth restriction, neonatal meningitis and respiratory distress (Table 6). Among the patients who have been registered into database, majority has had three neonatal/perinatal risk factors and 9 patients have had 5 or more risk factors (Table 7).

Table 6: Frequency of risk factors

\begin{tabular}{|l|c|}
\hline \multicolumn{1}{|c|}{ Risk Factor } & No. of patients \\
\hline Hypoglycaemia & 05 \\
\hline Neonatal jaundice & 30 \\
\hline Sepsis & 42 \\
\hline Meningitis & 23 \\
\hline RDS - Surfactant given & 09 \\
\hline RDS - CPAP given & 20 \\
\hline RDS - Ventilated & 12 \\
\hline RDS - Not ventilated & 08 \\
\hline IUGR/ low birth weight & 33 \\
\hline Birth asphyxia & 05 \\
\hline Meconium aspiration & 04 \\
\hline Electrolyte abnormalities & 03 \\
\hline
\end{tabular}

Table 7: Distribution of risk factors

\begin{tabular}{|l|c|}
\hline \multicolumn{1}{|c|}{ Number of risk factors } & $\mathbf{n}(\mathbf{\%})$ \\
\hline One risk factor only & $09(16.6)$ \\
\hline Two risk factors & $08(14.8)$ \\
\hline Three risk factors & $17(31.5)$ \\
\hline Four risk factors & $11(20.3)$ \\
\hline Five or more risk factors & $09(16.6)$ \\
\hline
\end{tabular}

Fifty patients have undergone an eye referral at an ophthalmology clinic and 40 of them have been reported as normal. Forty eight patients have been referred for an ear nose and throat (ENT) assessment by an ENT surgeon. Among the 25 patients who have had a 2-D echocardiogram, 21 had abnormal echo findings.

With the aid of this electronic database, a continuous record system for a single patient is maintained and relevant past information can be viewed and retrieved when taking future management steps. Also, progression of the therapies and results of improvement for individual patients can be detected. Therefore this is a useful tool for tailor made patient care.

\section{Discussion}

Paediatric MDT clinic at Colombo South Teaching Hospital failed to maintain a proper database management system previously and paper based data collection system caused difficulties in viewing and updating data, and usage of these data for designing future management plans and research purposes. A proper online electronic database management system can be designed by utilizing Google accounts and spreadsheets and a mobile app with user-friendly graphical user interface can be created for the database by utilizing AppSheet software. The mobile app can be created according the requirements of the clients.

The mobile app which was created for MDT clinic has three main tables/forms to enter data as personal data form, clinical data form and form to enter data of different MDT visits. This app enabled the team members to add, edit and view data of MDT patients. Telephone calls could be taken and patients' locations could be accessed through the app. Since database is accessible through phones, patients' past records could be reviewed and new records can be entered while conducting the clinic. Success of the therapies given to patients can be quantified by motor skills score and AIMS centile analysis. These spreadsheets could be linked to SPSS software to analyse data for research purposes. Even though this provides adequate confidentiality and security to data, all the authorized team members who share the app have an unrestricted access to add, edit or remove data.

Since the launching of the app and database from April 2019 as a pilot study for a one month period, records of 66 patients who are followed up at this MDT clinic were entered. Among them, majority were male patients and were recruited at birth for early neonatal intervention. Neonatal sepsis, intrauterine growth retardation (IUGR), neonatal jaundice and neonatal meningitis were the common risk factors identified. Majority of patients had 3 neonatal risk factors. A significant proportion of patients had undergone brain imaging, 2D echocardiography, ophthalmology, and ENT referral. This electronic database gives an online report of individual patient's clinical data and status/improvement of the clinical condition overtime, to the Multi-disciplinary team which is very effective to decide the future management plans. 
This method of creating an electronic database management system is practical to apply in most of the hospital and health care centers to design and maintain a proper record keeping system.

\section{Conclusions}

- Designing a clinical database management system is effective for patient management as well as audit and research purposes.

- Google spreadsheets and AppSheet software provide practical ways to generate a user friendly mobile app for clinical database management system.

- All members involved in patient care and research in the clinic have easy access to the database through internet that does not sacrifice security, quality and fidelity of clinical data.

\section{References}

1. Meineke FA, Staubert S, Lobe M, Winter A. A comprehensive clinical research database based on GDISG ODM and i2b2. Studies in Health Technology and Informatics 2014; 205:1115-9.
2. Rayhan RU, Zheng Y, Uddin E, Timbol C, Adewuyi o, Baraniuk JN. Administer and collect medical questionnaires with google documents: a simple, safe, and free system. Applied Medical Informatics 2013; 33:12-21.

3. Campbell FA, Ramey CT. Effects of early intervention on intellectual and academic achievement: A follow-up study of children from low-income families. Child Development, 1994; 65(2): 684-98.

https://doi.org/10.2307/1131410

4. Resnick MB, Eyler FD, Nelson RM, Eitzman DV, Bucciarelli RI. Developmental intervention for low birth weight infants: Improved early developmental outcomes. Pediatrics 1987; 80(1): 68-74 